\title{
Materials for a Rust-Flora of Riukiu Islands. II.*
}

\author{
By
}

\section{Naohide Hiratsuka.}

With 1 Text-figure.

Received September 10, 1940.

56. Hyalopsora Polypodii (Diet.) Magnus in Ber. Deutsch. Bot. Ges. XIX, p. 582, 1901.

Syn. Pucciniastrum Polypodii Dietel in Hedwigia, XXXVIII, p. (260), 1899.

On Diplazium oshimense H. Iто (Shikeshida). Okinawa Isl.: Motobumura (Kunigami-gun) (April 15, 1940, Y. TAIRA, no. 458). New to Riukiu Islands!

57. Coleosporium Evodiae Dietel in Ann. Myc. VII, p. 355, 1909.

On Evodia glauca Miq. (Shima-kuroki). Amami-ôshima 'Isl.: Sanbômura (Oct. 20, 1936, T. TAмотSU).

58. Hemileia Gardeniae-floridae SAwada in Transact. Nat. Hist. Soc. Formosa, XXI, p. 234, 1931 (nomen seminudum) ; Govern. Res. Inst. Formosa, Dept. Agrie. Rept. no. 61, p. 43 \& pl. I, figs. $27 \& 28,1933$ (nomen seminudum) ; Hiratsuka, f. \& Yoshinaga in Mem. Tottori Agric. Coll. III, p. 274,1935 .

On Gardenia jasminoides ELL. var. grandiflora NAKAI (Kuchinashi). Amami-ôshima Isl.: Tatsugô-mura (Oct. 15, 1936, T. TAmo'Tsu).

59. Skierka Agallocha Raciborski in Bull. Acad. Sci. Cracovie, Cl. Sci. math. et nat. (1909), p. 275, 1909; MaIns in Mycologia, XXXI, p. 188, 1939 ; Saccardo, Syll. Fung. XXI, p. 609 ; Sydow, Monogr. Ured. III, p. 332.

Soris uredosporiferis amphigenis, maculis minutis, flavidis vel flavobrunneolis insidentibus, sparsis, laxe aggregatis vel circinatim dispositis, rotundatis, minutissimis, $0.1-0.4 \mathrm{~mm}$ diam., diu epidermide tectis, flavobrunneis, brunneis vel atro-brunneis; uredosporis obovatis, oblongis, fusiformibus vel clavatis, aculeis brevibus crassis laxe aequaliterque obsitis,

* Continued from Vol. LIV, p. $166(1940)$. 
subhyalinis vel dilute flavis, $47-90 \times 20-43 \mu$, membranis tenuis, lateraliter $5-12 \mu$, formantibus juga, poris germinationis indistinctis.

Hab. On Excoecaria Agallocha L. var. genaina MüLL.-ARg. (Okinawajinkô, Shimashiraki). Okinawa Isl. : Nawa-shi (March 15, 1940, Y. TaIra, no. 398).

The present fungus coincides exactly to the description of Skierka Agallocha RACIBonski on Excoecaria Agallocha from Java in the characters of teleutospores. RACIBORSKI did not describe the uredostage of this fungus, which the writer takes pleasure in supplementary in this paper. This species is new to the mycological flora of our country.

60. Uromyces Tairae Hiratsuka, f. nov. spec.

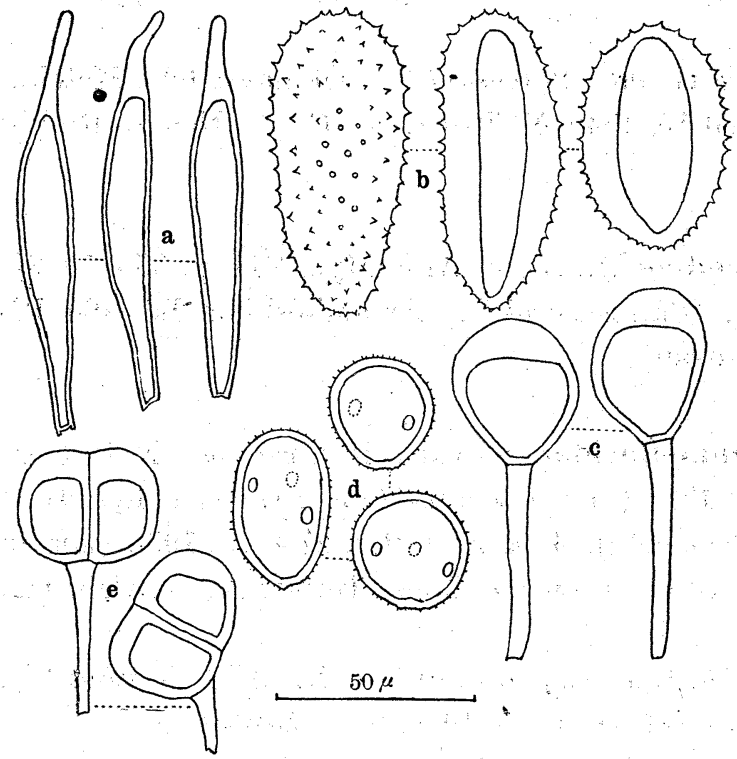

Text-fig. 1. a \& b. Skierka Agallocha RAC. on Excoecaria Agallocha var. genuina -a. Teleutospores; b. Uredospores. c \& d. Uromyces Tairae HIRATSUKA, f: on Messerschmidia argentea-a. Teleutospores. b. Uredospores. e. Teleutospores of Puccinia levis (SACC. et Brzz.) MAgNus on Digitaria Henryi.

Soris uredosporiferis amphigenis, sparsis, rotundatis vel irregularibus, minutis, mox nudis, pulverulentis, castaneobrunneis vel atro-brunneis ; uredosporis subglobosis, obovatis vel ellipsoideis, echinulatis, $22-30 \times 17-25 \mu$; episporio $1.5-2.4 \mu$ crasso, flavobrúnneis; pòris germinationis 3 (rarius 2 vel 4) sparsis praeditis. Teleutosporis immixtis, subglobosis vel obovatis, apice rotundatis, rarius etiam truncatis, valde incrassatis $(5-10 \mu)$, basi rotundatis: vel rarius attenuatis, levibus, brunneis, $\quad 25-38 \times 20-28 \mu$; pedicello persistenti, hyalino, usque $70 \mu$ longo.

Hab. On Messerschmidia argentea JoHN. (Monpa-no-ki) (Borraginaceae). Okinawa Isl.: Mabuni-mura (Shimajiri-gun) (March 2, 1940, Y. TAIRA, no. 372, type!).

Unfortunately teleutosori were not found and only a few teleutospores were present in the uredosori examined. 
61. Puccinia Absinthii (Hedw. f.) DE CANDolle, Fl. franç. VI, p. 56, 1815.

Syn. Uredo (Puccinia) Absinthii Hedwig, f. in Poir. in LAm., Encyel. Bot. VIII; p. 245, 1808.

On Artemisia dubia WALL. (Yomogi). Okinawa Isl.: Haneji-mura (Kunigami-gun) (April 8, 1940, Y. Taira, no. 424) ; Chinen-mura (Shimajiri-gun) (Jan. 20, 1940, Hiratsuka, f, no. 144).

On Artemisia japonica Thunb. (Otoko-yomogi).. Okinawa Isl. : Hanejimura (Kunigami-gun) (Jan. 15, 1940, Hiratsuka, f., no. 279). New to Riukiu Islands!

62. Puccinia anomala Rostrup in Thǜen in Flora, LXI, p. 92, 1878.

On Hordeum sativum JEśs. var: hexastichon Hack. ( $\hat{O}$-mugi) (cultivated). Amami-ôshima Isl.: Nase-machi (Dec. 10, 1929, T. Tамотsu). Okinawa Isl. : Haneji-mura (Kunigami-gun) (April 8, 1940, Y. TAIra, no. 428). New to Riukiu Islands!

63. Puccinia filipodia Cummins in Ann. Myc. XXXV, p. 98 \& textfig. 7, 1937.

On Heteropogon contortus Beauv. (Andropogon contortus L.) (Akahige-gaya). Okinawa Isl. : Urazoe-mura (Nakagami-gun) (March 13, 1940, Y. TAIRA, nos. $388 \& 389)$. Only uredospores are present in this collection. The present species is new to Japan.

64. Puccinia graminis Persoon in Neues Mag. Bot. I, p. 119, 1794 ; Syn. Fung. p. 228, 1801.

On Hordeum sativum Jess. var. hexastichon HACK. ( $\hat{O}$-mugi) (cultivated). Okinawa Isl. : Urazoe-mura (Nakagami-gun) (March 13, 1940, Y. TAIRA, no. 394).

On Triticum vulgare VILL. (Ko-mugi) (cultivated). Okinawa Isl.: Yontanzan-mura (Nakagami-gun) (March 8, 1940, Y. TAIrA, no. 384). New to Riukiu Islands!

65. Puccinia levigata Hiratsuka, f. in Hiratsuka, f. \& Yoshinaga in Mem. Tottori Agric. Coll. III, p. 315, 1935.

Syn. Diorchidium levigatum Sydow et Bulter in Ann. Mye. V, p. 500, 1907.

On Oplismenus compositus BeAuv. (Edauchi-chijimizasa). Okinawa Isl. : Shuri-shi (Nov. 27, 1898, Hiratsuka ; Feb. 19, 1940, Y. Taira, nos. 349 d" 478). 
66. Puccinia levis (Sacc. et Bizz.) Magnus in Ber.'Deutsch. Bot. Ges. IX, p. 190 \& pl. IX, figs. 28-37, 1891. 1882.

Sy̆n. Diorchidium leve Saccardo et Bizzizero in Michelia, II, p. 648,

On Digitaria Henryi Rendle (Mehishiba), Okinawa Isl. : Nishiharamura (Nakagami-gun) (Jan. 20, 1940, Hiratsuka, f., nos. 168, 169 \& 173). On Digitaria timorensis Balansa (Chimôru-mehishiba). Okinawa Isl. : Nishihara-mura (Nakagami-gun) (Jan. 20, 1940, Hiratsuka, f., no. 174).

This species is a new addition to the mycological flora of Japan.

67. Puccinia Miyoshiana Dietel in Engl. Bot. Jahrb. XXVII, p. $569 \&$ pl. VII, figs. 16 \& 17, 1899.

On Eccoilopus cotulifer A. Camus (Abura-susuki). Okinawa Isl.: Mt. Katsuu-dake (Kunigami-gun) (April 17, 1940, Y. TAIra, no. 464). New to Riukiu Islands !

68. Puccinia Phragmitis (Schum.) Körnicke-in Hedwigia, XV, p. $179,1876$.

Syn. Uredo Phragmitis Schumacher, Enum. PI. Saell. II, p. 231, 1803.

On Phragmites prostrata MaKino (Tsuru-yoshi). Okinawa Isl. : Tomigusuku-mura (Shimajiri-gun) (Feb. 24, 1940, Y. TAIRA, no. 363). New to Riukiu Islands!

69. Puccinia Polliniae-imberbis Hiratsuk, f. in Jour. Jap. Bot. XIII, p. 248 \& text-fig. 1, 1937.

Syn. Uredo Polliniae-imberbis Iто in Jour. Coll. Agric. Tohoku Imp. Univ. III, p. 246 \& pl. XII, fig. 11, 1909.

On Microstegium ciliatum A. CAmus var. Wallichianum Honda ( $\hat{O}$ sasagaya). Okinawa Isl.: Tomigusuku-mura (Shimajiri-gun) (Feb. 24, 1940, Y. TAIRA, no. 364). New to Riukiu Islands!

70. Puccinia Ploygoni-amphibii Persoon, Syn. Fung. p. 227, 1801.

On Pleuropterus multiflorus Turcz. (Tsuru-dokudami). Okinawa Isl.: Nawa-shi (March $24 \&$ May 21, 1940, Y. TAIra, nos. $413 \& 4480)$. New to Riukiu Islands !

71. Puccinia taiwaniana Hiratsuka, f. et Hashioka in Transact. Tottori Soc. Agric. Sci. VI, p. 240, 1935.

On Panicum patens L. (Hime-chigozasa). Okinawa Isl. : Haneji-mura (Kunigami-gun) (Jan. 13, 1940, Hiratsuka, f., nos. 94 d 95). New to Riukiu Islands! 
72. Aecidium Lysimachiae-japonicae Dietel in Engl. Bot. Jahrb. XXXIV, p. 590, 1905.

On Lysimachia japonica Thunb. (Ko-nasubi). Okinawa Isl. : Mt. Katsuu-dake (Kunigami-gun) (April 17, 1940, Y. TAIrA, no. 465); Motobumura (Kunigami-gun) (April 18, 1940, Y. TAIRA, no. 467). New to Riukiu Islands !

73. Uredo Alocasiae Sydow; Monogr. Ured. IV, p. 521, 1924.

On Alocasia macrorhiza Sснотт. (Kuwazu-imo). Okinawa Isl.: Shurishi (Jan. 10, 1940, Hiratsuka, f., nos. 2 \& 4) ; Nawa-shi (Jan. 22, 1940, Hiratsuka, f., nos. $325 \& 326)$. New to Riukiu Islands!

74. Uredo Arthraxonis-ciliaris Hennings in Hedwigia, XLVIII, p. 251, 1908.

On Arthraxon hispidus Makino (Kobunagusa). Okinawa Isl.: Kanegusuku-mura (Shimajiri-gun) (Jan. 21, 1940, Hrratsuka, f., no. 309); Ôsato-mura (Shimajiri-gun) (Jan. 20, 1940, Hiratsuka, f., no. 165) ; Takamine-mura (Shimajiri-gun) (Jan. 21, 1940, Hiratsuka, f., no. 192) ; Shurishi (March 25, 1940, Y. TAIRA; no. 451). New to Riukiu Islands!

75. Uredo Crepidis-integrae LindRoth in Acta Soc. pro Fauna et Flora fennica, XXII, no. 3, p. 11, 1902.

On Crepidiastrum lanceolatum NAKAI (Heranaren). Okinawa Isl.: Chinen-mura (Shimajiri-gun) (Jan. 20, 1940, Hiratsuka, f., nos. 145, 146 \& 147) ;Takamine-mura (March 19, 1940, Y. TAIrA, no. 405) ; Yontanzanmura (Nakagami-gun) (March 8, 1940, Y. TAIRA, no. 380). New to Riukiu Islands !

76. Uredo taiwaniana Hiratsuka, f. et Hashioka in Bot. Mag. Tokyo, XLVIII, p. 239, 1934.

On Epipremnum mirabile Scнотт. (Habu-kazura). Okinawa Isl.: Shuri-shi (March 12, 1940, Y. TaIRA, no. 397.). New to Riukiu Islands!

\section{Additional hosts.}

5. Phakopsora Artemisiae Hiratsuka, f.

On Artemisia japonica Thunb. (Otoko-yomogi). Okinawa Isl. : Urazoemura (Nakagami-gun) (March 13, 1940, Y. TAIrA, no. 390). 